\title{
ITERATION OF ANALYTIC MAPPINGS OF SEVERAL COMPLEX VARIABLES ${ }^{1}$
}

\author{
BY GIACOMO DELLA RICCIA
}

Communicated by W. T. Martin, October 18, 1968

Introduction. In this note we derive properties of the semigroup of iterates of an analytic mapping of several complex variables from general results on equicontinuous flows on locally compact metric spaces. These results are summarized in Theorems 1, 2, 3 and 4, the proofs of which are given by the present author elsewhere [1]. The main object of this note is Theorem 5 .

Let $T: X \rightarrow X$ be a continuous mapping of $X$ into itself. We indicate by $(X, T)$ the flow defined by the semigroup $\delta=\left[T^{n} ; n=0,1, \cdots\right]$ of iterates of $T$, where $T^{0}=$ identity mapping $I, T^{1}=T$ and $T^{m+n}$ $=T^{m} T^{n}=T^{n} T^{m}$. Given $x \in X, 0_{x}^{+}=\left\{y \in X \mid y=T^{n}(x)\right.$ for some nonnegative integer $n\}$ is the positive semiorbit of $x ; \overline{0}_{x}^{+}$is the closure in $X$ of $0_{x}^{+}$and the set of $\omega$-limit points of $x$ denoted by $\omega(x)$ is the set of limit points in $X$ of $0_{x}^{+}$. A nonempty closed subset $E \subset X$ is said to be minimal under $T$ if $x \in E \Rightarrow \overline{0}_{x}^{+}=E$.

RESULTS. In all the following we assume that $X$ is a locally compact metric space and that the semigroup $S$ is an equicontinuous sequence of functions on $X$.

THEOREM 1. If the set $\omega(x)$ of $\omega$-limit points of $x$ is nonempty, then it is minimal under $T$.

THEOREM 2. If the set $\omega(x)$ is nonempty, then $T$ is a homeomorphism of $\omega(x)$ onto itself and $\omega(x)$ is compact.

THEOREM 3. If $\Omega=U_{x \in X} \omega(x)$ is nonempty, then $T$ is a homeomorphism of $\Omega$ onto itself and $\Omega$ is a closed subset of $X$.

THEOREM 4. If we restrict $T$ and $T^{-1}$ to $\Omega$, then the semigroup $S^{-1}$ $=\left[T^{-n} ; n=0,1, \cdots\right]$ also is equicontinuous on $\Omega$ and on any compact subset $K \subset \Omega$ the group $\Gamma=\left[T^{ \pm n}, n=0,1, \cdots\right]$ is relatively compact in the topology of uniform convergence. If the sequence $\left[T^{n_{i}}\right]$ converges to $S$ on $K$, then $S$ is a homeomorphism of $K$ onto $S(K) \subset \Omega$ and $\left[T^{-n_{i}}\right]$ converges on $S(K)$ to the inverse $S^{-1}$ of $S$.

Now we consider flows $(D, T)$ where $T$ is an analytic mapping and

1 This research was supported by the National Aeronautics and Space Administration under grant NGR15-003-060. 
$D$ is a domain in the extended complex space $\bar{C}_{N}=\bar{C}_{1 \times} \cdots \times \bar{C}_{1}$ ( $N$ times), with $N \geqq 1$ and $\bar{C}_{1}$ being the extended complex plane. $T$ is defined by its $N$ components $\Phi_{j}(z), j=1, \cdots, N$ and $z \in D$, where the $\Phi_{j}$ 's are analytic functions, either holomorphic or meromorphic. However, in the meromorphic case we assume that the only singularities are poles, i.e. that the $\Phi_{j}$ 's have no points of indetermination in $D$.

We shall say that the flow $(D, T)$ is normal or, equivalently, that $S=\left[T^{n}\right]$ is normal if the sequence $\left[\Phi_{j}^{n} ; n=0,1, \ldots\right]$ of the $j$ th components of members of $S$ forms a normal family (in the sense of Montel) of analytic functions, for every $j=1, \cdots n$. It is known, even in the case of several complex variables [2], that a normal family is equicontinuous. We can thus apply the results mentioned above to normal flows and we want to show that in this case we have the following

Theorem 5. Let $(D, T)$ be a normal flow. If a sequence $\left[T^{n_{i}}\right]$ of iterates of $T$ converges to a nondegenerate mapping $S$, then

(1) the sequence $\left[T^{n_{i+1}-n_{i}}\right]$ converges to the identity mapping $I$.

(2) $T$ is a homeomorphism of $D$ onto itself.

(3) $S$ is a homeomorphism of $D$ onto itself.

(4) The limit of any other convergent subsequence of $\left[T^{n}\right]$ is always a homeomorphism of $D$ onto itself; more precisely the closure $\overline{\mathrm{S}}$ of $\mathrm{S}$ is a compact group of such homeomorphisms.

H. Cartan [3] proved parts (1), (2) and (3) in the special case where $D$ is a bounded domain. His proof is based on the fact that $T$ is holomorphic; it cannot be extended to unbounded domains and it cannot be used to prove part (4). Our method is essentially topological.

PRoof. We recall that a mapping is said to be nondegenerate if its Jacobian does not vanish identically on $D$ and that by convergence of a sequence of mappings we mean uniform convergence on compact subsets $K \subset D$.

Since $\lim _{i \rightarrow \infty} T^{n_{i}}=S$ is nondegenerate the set $D^{*} C D$ of interior points of $S(D)$ is not empty. Let us choose $x \in D^{*}$ and a sufficiently small compact neighborhood $\Sigma \subset D^{*}, x \in \Sigma$, such that $S(x)=y \in D^{*}$ and $S(\Sigma)=\Sigma^{\prime} \subset D^{*}$ is a compact neighborhood which contains $y$. Clearly $D^{*} \subset \Omega$. Since $\left[T^{n_{i}}\right]$ converges to $S$ on $\Sigma$ and, according to Theorem $4,\left[T^{-n_{i}}\right]$ converges to $S^{-1}$ on $\Sigma^{\prime}$, we see that the sequence $\left[T^{n_{i+1}-n_{i}}\right]$ converges to the identity mapping on $\Sigma$. But $\left[T^{n_{i+1}-n_{i}}\right]$ is a normal sequence; since it converges on an open subset of $D$, namely the interior of $\Sigma$, it converges everywhere on $D$. On the other hand, the limit of this sequence is an analytic mapping. Since this mapping 
coincides with the identity mapping on the interior of $\Sigma$, it follows that $\left[T^{n_{i+1}-n_{i}}\right]$ converges on $D$ to the identity mapping and we have proved part (1) of the theorem. Moreover, $\forall x \in D, \lim _{i \rightarrow \infty} T^{n_{i+1}-n_{i}}(x)$ $=x$ also means that $x \in D \Rightarrow x \in \omega(x)$. This shows that $D=\Omega$ $=\mathrm{U}_{x \in X} \omega(x)$. Hence part (2) of the theorem can be derived from Theorem 3. Since every $\omega(x)$ is a compact subset of $D$ (Theorem 2), it is clear that $S(D) \subset D$. According to Theorem $4, S$ is a local homeomorphism of $D$ onto $S(D)$; hence $S(D)=D^{*}$ is open in $D$. Furthermore, since each $\omega(x)$ is a minimal set (Theorem 1 ), it readily follows that $S(D)$ is dense in $D$. Consequently, the convergence of $\left[T^{-n_{i}}\right]$ on $S(D)$ to $S^{-1}$ can be extended to all of $D$ and $S^{-1}(D)=D$. This proves part (3) of the theorem. Finally, let us assume that there exists a sequence $\left[T^{p_{i}}\right]$ which converges to a degenerate mapping $Q$. Because of the compactness of every $\omega(x), Q(D) \subset D$. But now $Q(D)$ is a closed nowhere dense subset of $D$. Let us then choose $x \notin Q(D)$ and consider $\lim _{i \rightarrow \infty} T^{p_{i}}(x)=y \in Q(D)$. Clearly $\omega(y) \subset Q(D)$ and therefore $x \notin \omega(y)$. But, on the other hand, we know that $x \in \omega(x)$ and since $y \in \omega(x)$ and $\omega(x)$ is minimal, we must have $\omega(y)=\omega(x)$; hence $x \in \omega(y)$. This contradiction shows that $Q$ is nondegenerate and, consequently, that it is a homeomorphism of $D$. This completes the proof of the theorem.

Remark. P. Fatou [4], in his work on the iteration of rational functions $R(z)$ of one complex variable, conjectured that for some domains $D$, called singular domains, some sequences of iterates of $R$ converge to homeomorphisms of $D$ onto itself, and some others might converge to a degenerate mapping which maps $D$ onto a point of the boundary of $D$. Part (4) of Theorem 5 shows that the conjecture is false: on a singular domain all limits are homeomorphisms.

\section{REFERENCES}

1. G. Della Riccia, Equicontinuous flows (semi-groups) on locally compact or complete metric spaces, Math Systems Theory (to appear).

2. G. Julia, Sur les familles de fonctions analytiques de plusieurs variables, Acta Math. 47 (1926), 53.

3. H. Cartan, Sur les fonctions de plusieurs variables complexes. L'itération des transformations intérieures, d'un domaine borné, Math. Z. 35 (1932), 760.

4. P. Fatou, Sur les équations fonctionnelles, Bull. Soc. Math. France 48 (1920), 33. See, in particular, pp. 57-58.

INDIANA UNIVERSITY, BLOOMINGTON, INDIANA 47401 